Review

\title{
Multi-Step Usage of in Vivo Models During Rational Drug Design and Discovery
}

\section{Charles H. Williams ${ }^{1}$ and Charles C. Hong ${ }^{1,2, *}$}

1 Division of Cardiovascular Medicine, Department of Medicine, Vanderbilt University School of Medicine, Nashville, TN 37232, USA; E-Mail: Charles.h.williams@ vanderbilt.edu

* Author to whom correspondence should be addressed; E-Mail: Charles.c.hong@ vanderbilt.edu; Tel.: +1-615-936-7032.

Received: 1 March 2011; in revised form: 25 March 2011 / Accepted: 29 March 2011 / Published: 1 April 2011

\begin{abstract}
In this article we propose a systematic development method for rational drug design while reviewing paradigms in industry, emerging techniques and technologies in the field. Although the process of drug development today has been accelerated by emergence of computational methodologies, it is a herculean challenge requiring exorbitant resources; and often fails to yield clinically viable results. The current paradigm of target based drug design is often misguided and tends to yield compounds that have poor absorption, distribution, metabolism, and excretion, toxicology (ADMET) properties. Therefore, an in vivo organism based approach allowing for a multidisciplinary inquiry into potent and selective molecules is an excellent place to begin rational drug design. We will review how organisms like the zebrafish and Caenorhabditis elegans can not only be starting points, but can be used at various steps of the drug development process from target identification to pre-clinical trial models. This systems biology based approach paired with the power of computational biology; genetics and developmental biology provide a methodological framework to avoid the pitfalls of traditional target based drug design.
\end{abstract}

Keywords: phenotypic screen; drug discovery; small molecules; drug design chemical genetics; model organisms 


\section{Introduction}

Cancer, Alzheimer, diabetes; all are leading causes of death in the US. Unlike exogenous factors like HIV/AIDS and influenza, they are the result of endogenous developmental programming behaving in an aberrant fashion. As the average lifespan of human increases, certain biological machineries in our bodies start to break down. Even if all exogenous infections were obliterated from the face of the globe we would still face these diseases. Pharmaceutical companies face the challenge of modulating these developmental processes with small molecules. The human genome consists of approximately 25,000 genes [1]. Of these genes only 3000 of which are thought to be druggable and $50 \%$ of those thought to be disease causing [2]. A list of $\sim 1500$ potentially druggable disease causing biomolecules is now the center of focus in the pharmaceutical industry. The current paradigm of drug design revolves around these biomolecule targets and designing and identifying small molecules that modulate the activity of them in vitro or in silico; this is called a "target centered" approach (Figure 1a). Let us look at the track record of this approach. Only 1 of 5000 discoveries makes it to market from the bench side. The average time it takes a drug to reach the bedside from discovery is 12 years, and a single pharmaceutical agent costs from 500 million to 2 billion dollars to bring to market [3]. How could all but one of 5000 discoveries end up being useless as a therapeutic? The answer may lie in the "target centered" paradigm that has driven drug design for the past 50 years.

Figure 1. Workflow for two paradigms of drug discovery. (A) Conventional "Target centered" drug discovery; (B) In vivo model based drug discovery.

(A)
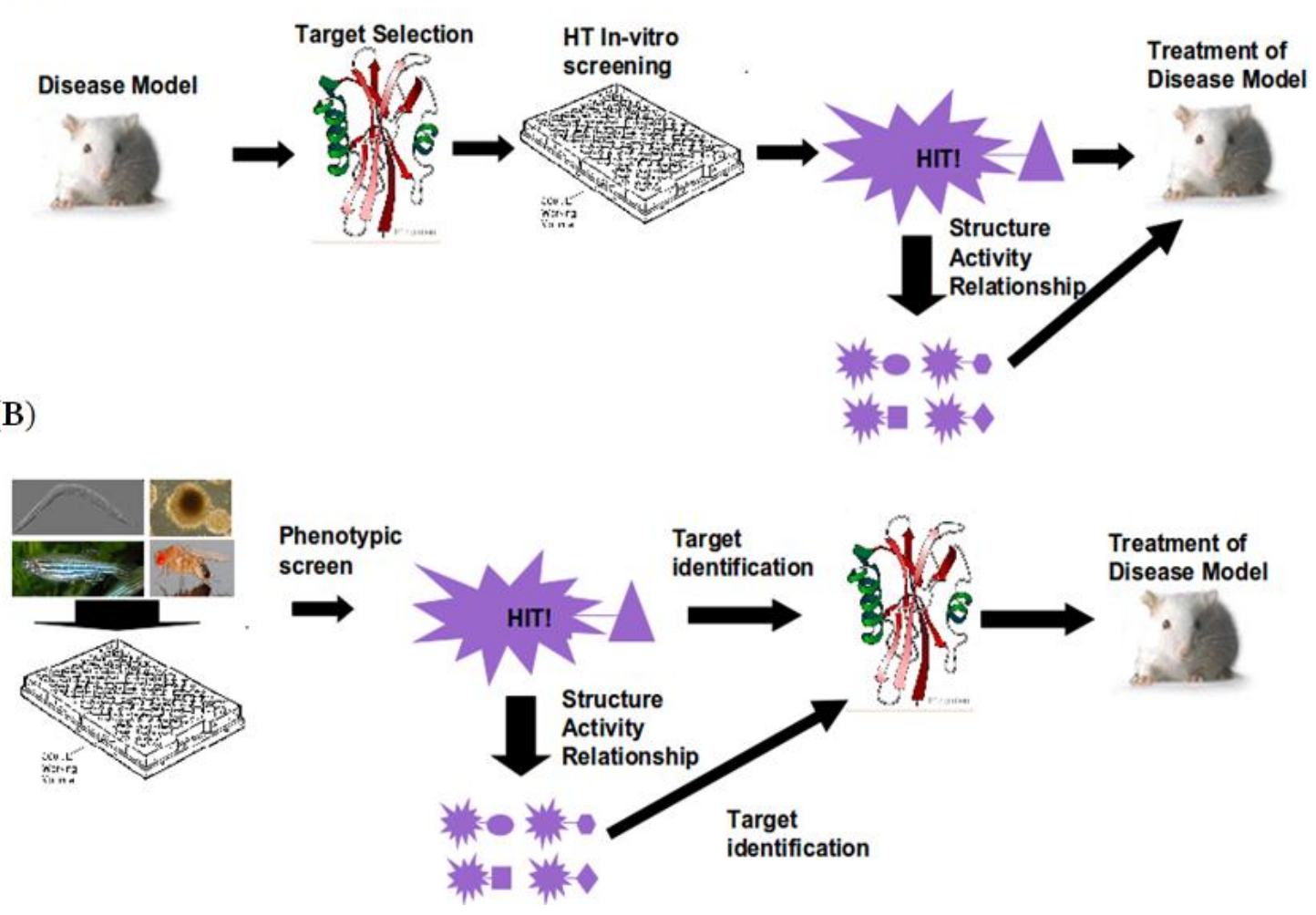

This paradigm is not without its successes. The anti-hypertensive agent Captopril produced by Bristol Meyer-Squibb is a potent and reversible inhibitor of Angiotensin-converting enzyme. 
Approved by the FDA in 1981 it rapidly became an effective treatment for hypertension and heart failure [4]. And Novartis produced an FDA approved drug Aliskiren, in 2007 with a combination of $\mathrm{X}$-ray crystallography and computer aided design [5]. However, most of these target centric designs often fail to meet the standard when ADMET (absorption, digestion, metabolism and toxicity) is evaluated after years of research and millions of dollars. In place of this paradigm, a systems biology approach is emerging using a phenotypic screen that inherently takes into account certain ADMET properties. The following article will present a systematized development method for rational drug design based on phenotype driven discovery.

\section{Workflow}

The first step in in vivo phenotypic discovery is the defining the desired phenotype of a "hit" compound. Currently, there are two major types of phenotypic screens. First is a forward chemical genetic screen, which consists of inducing a desired phenotype in a wild typesetting in your model organism (Figure 1B). The second is a therapeutic screen, taking a disease model and reversing it with a compound. However, before either screen can be done a decision must be made about which model system to use. There is also an emerging third type of screen, known as a pathway screen. This screen looks for a change in a particular signaling pathway in vivo. The second decision that must be made is which model will be used for the screen. The model organisms are, namely, Drosophila, C. elegans, zebrafish, or stem cells, which are all discussed below and summarized (Table 1). Further, strategies to identify the molecular target of the hit ligand must be considered; since a compound with completely unknown mechanism of action is unlikely to gain ready acceptance. Various techniques for target identification exist and are not mutually exclusive. Broadly, they are transcriptome profiling, affinity pull down, affinity response target screening, and yeast 3 hybrid screening. At this juncture, it is important to consider whether a "hit" compound is bioactive in live animals, depending on what model was used for initial screening. Assuming the ultimate goal of a chemical screen is to discover novel therapeutics for humans, it would be important to test whether the small molecule intervention robustly elicits desired effects without toxicities in inexpensive model organisms, prior to advancing the small molecule toward much more expensive clinical testing. Indeed, it has been estimated that just $10 \%$ improvements in predicting failures before clinical trials could save \$100 million per drug [6].

Table 1. Comparison of in vivo small molecule discovery models.

\begin{tabular}{ccccc}
\hline & C. elegans & D. melanogaster & D. rerio & M. musculus \\
\hline Generation Time & $3-5$ days & $10-14$ days & $3-4$ months & $6-8$ weeks \\
Media & Solid or liquid & Solid & Liquid & N/A \\
Ease of Obtaining Embryos & +++++ & +++++ & ++++ & N/A \\
Number of Genes & $\sim 19,000$ & $\sim 13,000$ & $\sim 25,000$ & $\sim 25,000$ \\
Homology to Human Genome & $>50 \%$ & $>60 \%$ & $>70 \%$ & $>90 \%$ \\
Annual Cost & + & + & ++ & ++++ \\
\hline
\end{tabular}




\section{Organismal Models}

Invertebrates are small, low cost and tend to have high fecundity; as such, they are one possible avenue for screening models. One such invertebrate worming its way into drug discovery is C. elegans; it is a nematode with a short life cycle (approximately 3.5 days) and can be raised in liquid media while consuming E. coli. Each adult hermaphrodite consists of 959 cells and can produce 300 larvae by self-fertilization. Since it started being used by Sydney Brenner in 1960s [7], it has been used to study cancer [8], neuronal cell death [9], and cilia [10]. C. elegans has also been established as a disease model of Alzheimer's disease [11], Parkinson's disease [12], Friedrich ataxia [13], and diabetes mellitus [14]. Recently the small nematode has made progress as a screening tool, in part due to a HTS method of arraying larvae [15]. For example, Kwok and colleagues identified a novel calcium antagonist that targets egl-19, the L-type calcium channel alpha1-subunit [16]. Additionally, an automated image analysis based high-throughput screen utilizing transgenic worms identified known autophagy enhancers that could be used to treat human liver diseases caused by protein misfolding [17]. Despite the versatility of $C$. elegans as a screening model, it has a few important drawbacks. First, its homology to the human genome is relatively low, with approximately $50 \%$ of human genes having orthologues [18]. Many organs in the human body do not have corresponding structures in $C$. elegans. The $C$. elegans body is also covered by a thick cuticle that is hard to penetrate [19]. Many compounds will not penetrate it unless a special solvent containing naphthalene and para-dichlorobenzene is added to the media [20]. Overall, C. elegans is promising model for in vivo small molecule screening to yield tools for simple biologically conserved pathways.

Table 2. Summary of types of phenotypic screens in zebrafish.

\begin{tabular}{|c|c|c|}
\hline Embryo & Type of Screen & Readout \\
\hline Wild type & Chemical genetic & Morphological defect \\
\hline $\begin{array}{c}\text { Disease model } \\
\text { (eg. Blood pooling) }\end{array}$ & Therapeutic & Restored to wild type \\
\hline $\begin{array}{l}\text { Trangenic Embryo } \\
\text { Tg(Flk:dsRed })\end{array}$ & Transgene assisted & $\begin{array}{l}\text { Altered anatomy visualızed } \\
\text { through transgenic marker }\end{array}$ \\
\hline $\begin{array}{c}\text { Transgenic Reporter Line } \\
\text { Tg(Top:dGFP) }\end{array}$ & $\begin{array}{l}\text { Pathway Reporter } \\
\text { inhibitor /enhancer }\end{array}$ & Down regulated Reporter gene \\
\hline
\end{tabular}


Another invertebrate that shows potential for small molecule screening is the fruit fly Drosophila melanogaster. It has been used for over a century in genetics research. Drosophila is anatomically more similar to mammals and has more than $60 \%$ genomic homology to humans [21]. As with C. elegans there are many mutants and even a fair number of disease models ranging from muscular dystrophy [22], to Alzheimers [23]. Recently this model has successfully been used by Chang and colleagues in a therapeutic screen. Briefly, a screen of 2000 compounds resulted in identification of nine molecules that rescue the Drosophila model of fragile X syndrome [24]. This screen, in addition to providing new small molecule tools, uncovered a novel function for muscarinic cholinergic receptors acting in parallel to the GABAergic pathway in rescuing fragile $\mathrm{X}$ syndrome phenotypes. Despite these impressive results, the drawbacks of using Drosophila as a small molecule screening model are three-fold. First, the organism is covered with a cuticle that is hard to penetrate. Secondly, Drosophila does not grow in a liquid media so precise dosing of small molecules in a high throughput manner is difficult. Thirdly, as with $C$. elegans, the Drosophila model lacks some anatomical (e.g., closed circulatory system) and genetic components that are present in humans.

As a vertebrate model of human disease, mouse has long been the gold standard. However, the size, labor and time requirements for mice make them cost prohibitive for conducting small molecule screens. However, there has been some limited success in chemical screening using mice. A proof of principle comparison of anti-TB drugs has been conducted and shows promise as a methodology for conduction large scale screens for TB therapeutics [25]. In recent years, another vertebrate model has emerged at the forefront of small molecule screening. Over the past 20 years the zebrafish has made a substantial impact in biological research. It has been used to study multiple areas ranging from vascular development [26,27] and neural development [28], to disease models for cancer [29] and melanocyte development [30]. The embryos are roughly $1 \mathrm{~mm}$ in size and 3-6 embryos can comfortably be arrayed in the wells of a 96-well plate [31]. Its size, low cost, and fecundity make zebrafish an attractive model for basic research. In addition, the liquid media, genetic homology to humans (over 70\%), and rapid development of most organs within $48 \mathrm{~h}$ post fertilization, make it an ideal model for small molecule screening. With these advantages, the small teleost is swimming its way into the field of chemical genetics. There are four major models for phenotypic screening in zebrafish; they are chemical genetic, therapeutic, transgene assisted and pathway based screens (Table 2). We have, in a chemical genetic screen, used perturbation of dorso-ventral (front to back) polarity in zebrafish embryos to discover dorsomorphin, the first selective Bone morphogenic protein (BMP) type I receptor [32]. Dorso-ventral patterning in zebrafish is established primarily through a functional antagonism between Wnt and BMP, but perturbations in numerous other pathways can distort the overall embryonic patterning. In a slightly more focused, transgene assisted, approach screening for anti-angiogenic agents, transgenic fish expressing GFP under a vasculature specific promoter were used in an automated and quantitative screen. This identified two known anti-angiogenic agents along with one other novel compound [33]. Zebrafish have also been used to screen for modifiers of specific pathways such as Fgf through the use of a florescent reporter [34].

In addition to organism based phenotypic screens, chemical screen using pluripotent stem cells has emerged as a viable alternative. A major benefit of screening in human derived stem cells is that one doesn't need to worry about translatability in terms of conservation and orthology. Stem cells are grown in liquid media and can be arrayed in a 96-well format to form uniform sized embryoid 
bodies [35]. Stem cells can give rise to all three germ layers and any cell type should be derived. Small molecules have successfully been used to create numerous cell types including cardiomyocytes [36,37]. Because of this attractive therapeutic potential, for directed differentiation, a small molecule screens using stem cells (R1 cells) was conducted by Zhu and colleagues for small molecules that promoted differentiation into definitive endoderm by assaying for expression of a sox17-rfp [38]. Additionally, ectoderm derived neurospheres were also used for assay for neurogenic compounds in a phenotypic screen [39]. However, as with many cell based assays this model for phenotypic screening could also potentially lead to identification of compounds that have poor ADME properties. This methodology could be applied to the generation of any number of cell types for which specific markers are available, such as Beta-cells and could substantially help the field of regenerative medicine.

Table 3. Overview of molecular target identification technologies.

\begin{tabular}{lll}
\hline Strategy & Advantages & Disadvantages \\
\hline $\begin{array}{l}\text { Affinity } \\
\text { Chromatography }\end{array}$ & Traditionally used and readily accepted & Requires sophisticated equipment \\
& & Low throughput \\
Expression Profiling & No chemical modifications & Requires chemical modification \\
& High throughput & Data can be noisy \\
& & Requires sophisticated bioinformatics \\
Yeast 3 Hybrid & High throughput & Imprecise \\
& & Non native environment \\
& & Not suitable for membrane bound proteins \\
& & Requires chemical modifications \\
DARTS & No chemical modifications & Low throughput \\
& Does not require high affinity & \\
\hline
\end{tabular}

\section{Target Identification}

Traditional drug design starts with the identification of a possible therapeutic target. For phenotypic screens target identification is the bottle neck for drug development. However, major advances are being made in the field for more efficient and rapid identification. These techniques can for our purposes be broken into two broad categories; techniques requiring modification of the small molecule and techniques that can use the native molecule.

The first two methods, that don't require modification of the ligand, are based on comparative transcriptome profiling. By utilizing a network systems biology approach one can identify the central nodes of affected gene clusters. This can yield a broad view of the mechanism of action [40]. One can also use hierarchical clustering which has been successful in both yeast and rat tissue [41]. This however provides documentable cell-type specific effects, and also microarray specific effects. An alternative approach is through the use of the Connectivity Map [42]. This takes a rank based pattern matching strategy applied to a database of over 7000 expression profiles representing 1309 compounds to identify similarities and thus potential target pathways [43].The problem with the listed techniques thus far, has been that they are unable to pinpoint specific binding partners. There is a new technique called Drug Affinity Response Target Screening (DARTS) [44]. When a small molecule binds a molecule there is a physical response. This response can be proteolytically protective, and the resultant 
protected peptide sequence can yield the target protein. The efficacy of this method has been tested with both high and low binding affinity molecules. This technique does not depend on an in vivo response and gives large amount of flexibility.

If one has a good understanding of the structure activity relationship for the compound and the phenotype one can alter the small molecule so it can be affixed to a linker. Once a linker is affixed to the small molecule, there are two approaches that can be taken. The first is the chemical proteomic approach; this approach is based on affinity chromatography and utilizes the pull down of candidate proteins. This has been the golden standard in the field for many years. With advances in MS technology and protein technologies there are new flavors of this approach that use isotope labeled proteins (a significant review of these methods was written by Rix and Superti-Furga [45]). Another technique that can be used is a variation of the yeast two hybrid screen. The yeast 3 hybrid screen requires the small molecule to be linked to a methotrexate moiety (the anchor). This molecule will attach itself to the hybrid dihydrofolate reductase-LexA DNA binding domain via the methotrexate. The small molecule bait will bring any target molecules (from a cDNA library of Gal4 activation domain fusions) within range of the DNA binding domain to elicit a His-3 reporter [46]. This is a versatile system and the cDNA libraries are commercially available from a number of sources for a number of different model systems.

\section{SAR and Compound Optimization}

Most SAR studies are done with a priori knowledge of the target molecule or pathway. Recent advances in computer technology have made molecular modeling based on crystallized protein structure feasible for even small laboratories [47]. Traditionally, an in vitro assay is used for testing analogs to determine SAR. However, it is possible to conduct SAR in vivo using a phenotypic model as a read out. Hao and colleagues used the zebrafish model to perform the first in vivo SAR of dorsomorphin analogues. With this system they were able to derive compounds with differing specificities for Vegf and Bmp receptors [48]. Although the target for dorsomorphin was known, this new paradigm of in vivo SAR could be used prior to any knowledge of target pathways as it only requires a simple read-out. This in vivo approach also helps in identifying not just the most potent forms of the compound; but also takes into account the in vivo permeability of the compound.

\section{Vertebrate Toxicity}

One of the major reasons potential drugs fail before they reach the market is because of the off target effects that manifest during clinical trials. The International Conference on Harmonization (ICH) S7A guidelines state that prior to clinical trial in humans, that pharmacophores must be evaluate on the vital functions, namely the circulatory system, CNS, G.I., and skeletal systems [49]. Of the models discussed above, only the zebrafish and mouse have all these systems (Figure 2). Traditionally, toxicological data is obtained from mouse. This is however, as mentioned before, a costly model system. In recent years zebrafish have emerged as a viable, low cost alternative for determining compound affects on these major organ systems and could be a useful tool for identifying liabilities of pharmacophores during early stages of drug development. 
Figure 2. Conservation of organ systems between zebrafish and humans. The zebrafish is a versatile model that is useful not only drug discovery but rapid development of organ systems makes it ideal for assessing biochemical safety and toxicity

\begin{tabular}{|c|}
\hline CNS \\
Cardiovascular \\
system \\
Liver \\
Pancreas \\
Gall bladder \\
Intestine \\
\hline
\end{tabular}
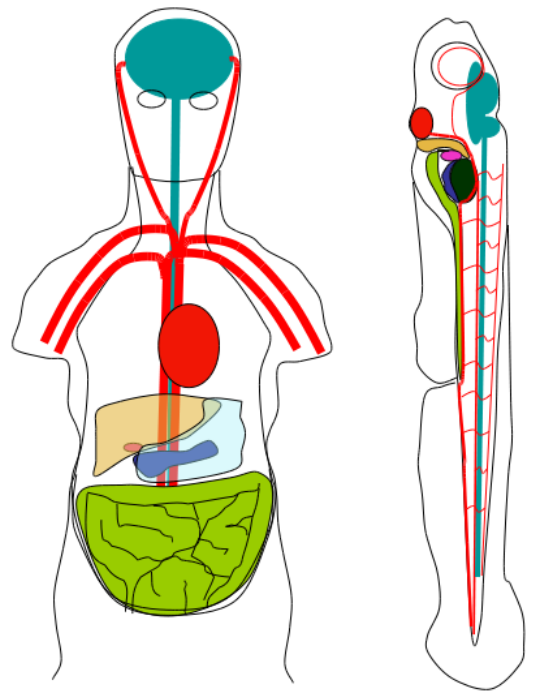

The zebrafish circulatory system forms and is functional by $23 \mathrm{~h}$ post fertilization (hpf), and by $48 \mathrm{hpf}$ the heart has undergone looping to form a distinct atrium and ventricle. The transparency of the zebrafish embryo allows for easy visualization of both the heart and blood circulation. A screen was done with 23 drugs known to cause QT prolongation and torsades de pointes in man, to identify if zebrafish would phenocopy the results in 3 days post fertilization (dpf) zebrafish embryos. The results identified 18 that caused brachycardia [50]. Furthermore, a transgenic line that expresses GFP under a cardiac specific promoter has been developed and an automated method of determining heart rate in a high throughput manner has been developed [51]. For further cardiovascular effects, zebrafish blood can be visualized for hemorrhages and an image capture analysis of single erythrocytes can be conducted to assess the contractility of the heart [52]. Within the first $48 \mathrm{~h}$ post fertilization zebrafish develop a touch response, at $68 \mathrm{hpf}$ they have a visual startle response, are capable of free swimming at $96 \mathrm{hpf}$, and develop an auditory startle response by $5 \mathrm{dpf}$. Screens have been conducted on all of these nervous system responses and have shown remarkable predictability of identifying problems also caused in humans [53-56]. For example, in a study of 8 compounds that can cause visual impairment in man, 6 inhibited the optokinetic motor response correctly in zebrafish [57]. At 36 hpf the zebrafish digestive tract begins to form, and it becomes fully functional and zebrafish are fed exogenously by $5 \mathrm{dpf}$ [58]. As with blood cells, the optical clarity of the zebrafish allows for easy visualization of the peristaltic contractions of the intestine. In a study of compound effects on gut mobility 8 of 10 compounds showed corresponding decreased contractility in zebrafish [58]. Finally, as a vertebrate the zebrafish model allows one to assess bone mineralization by $10 \mathrm{dpf}$. This is a proof of principle as the study was conducted with prednisolone which substantially decreased bone mineralization in zebrafish [59]. The flexibility and anatomical conservation between man and zebrafish make it ideal for assessing toxicity of various organ systems. 


\section{Afterword}

Rational drug design has traditionally depended on a thorough understanding of the disease to be treated so a target protein can be selected and ligands can be screened against that molecular target. However, many diseases are complex, and regardless of the progress made in understanding the disease a coherent model cannot be established. This is particularly true for psychological and neurological diseases such as Alzheimer's and Parkinson's disease. In the treatment of many psychological disorders the only pharmaceuticals available target serotonin, dopamine, or norepinephrine signaling. These diseases are diagnosed based on behavior; therefore a model with quantifiable behaviors is necessary for in vivo screening. Recently, a screen for chemical modulators of wake/rest cycle was conducted using zebrafish [60]. This study implicated a novel pathway involving ERG (ether-a-go-go) potassium channel proteins. Another potential avenue for in vivo screening is cancer. Zebrafish can be injected with human cancer cells at $2 \mathrm{dpf}$ and assessed for both angiogenic response (1 day post implantation) and metastatic behavior (5 days post implantation) [61]. Conducting a small molecule screen on these fish could yield novel therapeutics that target the cancer cells specifically in an in vivo environment. Furthermore different cancer cell lines respond differently and as such this screen could be conducted on different lines of cancer cells and yield different results. Whether a disease is well understood or not, phenotypic screens using in vivo small animal models like the zebrafish show great promise for aiding drug discovery and development at many steps of the drug development process.

\section{References}

1. International Human Genome Sequencing Consortium. Finishing the euchromatic sequence of the human genome. Nature 2004, 431, 931-945.

2. Hopkins, A.L.; Groom, C.R. The druggable genome. Nat. Rev. Drug Discov. 2002, 1, 727-730.

3. Adams, C.P.; Brantner, V.V. Spending on new drug development. Health Econ. 2010, 19, 130-141.

4. Talele, T.T.; Khedkar, S.A.; Rigby, A.C. Successful applications of computer aided drug discovery: Moving drugs from concept to the clinic. Curr. Top Med. Chem. 2010, 10, 127-141.

5. Zon, L.I.; Peterson, R.T. In vivo drug discovery in the zebrafish. Nat. Rev. Drug Discov. 2005, 4, 35-44.

6. Cohen, N.C. Structure-based drug design and the discovery of aliskiren (Tekturna): Perseverance and creativity to overcome a R\&D pipeline challenge. Chem. Biol. Drug Des. 2007, 70, 557-565.

7. Brenner, S. In the beginning was the worm. Genetics 2009, 182, 413-415.

8. Poulin, G.; Nandakumar, R.; Ahringer, J. Genome-wide RNAi screens in Caenorhabditis elegans: Impact on cancer research. Oncogene 2004, 23, 8340-8345.

9. Putcha, G.V.; Johnson, E.M. Men are but worms: Neuronal cell death in C. elegans and vertebrates. Cell Death Differ. 2004, 11, 38-48.

10. Smith, K.R.; Kieserman, E.K.; Wang, P.I.; Basten, S.G.; Giles, R.H.; Marcotte, E.M.; Wallingford, J.B. A role for central spindle proteins in cilia structure and function. Cytoskeleton 2010, 68, 112-124. 
11. Ewald, C.Y.; Li, C. Understanding the molecular basis of Alzheimer's disease using a Caenorhabditis elegans model system. Brain Struct. Funct. 2010, 214, 263-283.

12. Harrington, A.J.; Hamamichi, S.; Caldwell, G.A.; Caldwell, K.A. C. elegans as a model organism to investigate molecular pathways involved with Parkinson's disease. Dev. Dyn. 2010, 239, 1282-1295.

13. Puccio, H. Multicellular models of Friedreich ataxia. J. Neurol. 2009, 256 (Suppl. 1), 18-24.

14. Morcos, M.; Hutter, H. The model Caenorhabditis elegans in diabetes mellitus and Alzheimer's disease. J. Alzheimers Dis. 2009, 16, 897-908.

15. Burns, A.R.; Kwok, T.C.Y.; Howard, A.; Houston, E.; Johanson, K.; Chan, A.; Cutler, S.R.; McCourt, P.; Roy, P.J. High-throughput screening of small molecules for bioactivity and target identification in Caenorhabditis elegans. Nat. Protoc. 2006, 1, 1906-1914.

16. Kwok, T.C.Y.; Ricker, N.; Fraser, R.; Chan, A.W.; Burns, A.; Stanley, E.F.; McCourt, P.; Cutler, S.R.; Roy, P.J. A small-molecule screen in C. elegans yields a new calcium channel antagonist. Nature 2006, 441, 91-95.

17. Gosai, S.J.; Kwak, J.H.; Luke, C.J.; Long, O.S.; King, D.E.; Kovatch, K.J.; Johnston, P.A.; Shun, T.Y.; Lazo, J.S.; Perlmutter, D.H.; et al. Automated high-content live animal drug screening using $C$. elegans expressing the aggregation prone serpin $\alpha 1$-antitrypsin Z. PLoS One 2010, 5, e15460.

18. Harris, T.W.; Chen, N.; Cunningham, F.; Tello-Ruiz, M.; Antoshechkin, I.; Bastiani, C.; Bieri, T.; Blasiar, D.; Bradnam, K.; Chan, J.; et al. WormBase: A multi-species resource for nematode biology and genomics. Nucleic Acids Res. 2004, 32 (Suppl. 1), D411-D417.

19. Wells, W.A. High-throughput worms. NemaPharm, Inc. Chem. Biol. 1998, 5, R147-R148.

20. Kokel, D.; Li, Y.; Qin, J.; Xue, D. The nongenotoxic carcinogens naphthalene and para-dichlorobenzene suppress apoptosis in Caenorhabditis elegans. Nat. Chem. Biol. 2006, 2, 338-345.

21. Bier, E. Drosophila, the golden bug, emerges as a tool for human genetics. Nat. Rev Genet. 2005, 6, 9-23.

22. van der Plas, M.C.; Pilgram, G.S.K.; de Jong, A.W.; Bansraj, M.R.; Fradkin, L.G.; Noordermeer, J.N. Drosophila Dystrophin is required for integrity of the musculature. Mech. Dev. 2007, 124, 617-630.

23. Nichols, C.D. Drosophila melanogaster neurobiology, neuropharmacology, and how the fly can inform central nervous system drug discovery. Pharmacol. Ther. 2006, 112, 677-700.

24. Chang, S.; Bray, S.M.; Li, Z.; Zarnescu, D.C.; He, C.; Jin, P; Warren, S.T. Identification of small molecules rescuing fragile X syndrome phenotypes in Drosophila. Nat. Chem. Biol. 2008, 4, 256-263.

25. Nikonenko, B.V.; Samala, R.; Einck, L.; Nacy, C.A. Rapid, simple in vivo screen for new drugs active against Mycobacterium tuberculosis. Antimicrob. Agents Chemother. 2004, 48, 4550-4555.

26. Williams, C.; Kim, S.; Ni, T.T.; Mitchell, L.; Ro, H.; Penn, J.S.; Baldwin, S.H.; Solnica-Krezel, L.; Zhong, T.P. Hedgehog signaling induces arterial endothelial cell formation by repressing venous cell fate. Dev. Biol. 2010, 341, 196-204.

27. Hong, C.C.; Kume, T.; Peterson, R.T. Role of crosstalk between phosphatidylinositol 3-kinase and extracellular signal-regulated kinase/mitogen-activated protein kinase pathways in artery-vein specification. Circ. Res. 2008, 103, 573-579.

28. Takada, N.; Kucenas, S.; Appel, B. Sox10 is necessary for oligodendrocyte survival following axon wrapping. Glia 2010, 58, 996-1006. 
29. Goessling, W.; North, T.E.; Zon, L.I. New waves of discovery: Modeling cancer in zebrafish. J. Clin. Oncol. 2007, 25, 2473-2479.

30. Richardson, J.; Lundegaard, P.R.; Reynolds, N.L.; Dorin, J.R.; Porteous, D.J.; Jackson, I.J.; Patton, E.E. Mc1r Pathway regulation of zebrafish melanosome dispersion. Zebrafish 2008, $5,289-295$.

31. Hao, J.; Williams, C.H.; Webb, M.E.; Hong, C.C. Large scale zebrafish-based in vivo small molecule screen. J. Vis. Exp. 2010, 46, 2243.

32. Yu, P.B.; Hong, C.C.; Sachidanandan, C.; Babitt, J.L.; Deng, D.Y.; Hoyng, S.A.; Lin, H.Y.; Bloch, K.D.; Peterson, R.T. Dorsomorphin inhibits BMP signals required for embryogenesis and iron metabolism. Nat. Chem. Biol. 2008, 4, 33-41.

33. Tran, T.C.; Sneed, B.; Haider, J.; Blavo, D.; White, A.; Aiyejorun, T.; Baranowski, T.C.; Rubinstein, A.L.; Doan, T.N.; Dingledine, R.; et al. Automated, quantitative screening assay for antiangiogenic compounds using transgenic zebrafish. Cancer Res. 2007, 67, 11386-11392.

34. Molina, G.; Vogt, A.; Bakan, A.; Dai, W.X.; de Oliveira, P.Q.; Znosko, W.; Smithgall, T.E.; Bahar, I.B.; Lazo, J.S.; Day, B.W.; et al. Zebrafish chemical screening reveals an inhibitor of Dusp6 that expands cardiac cell lineages. Nat. Chem. Biol. 2009, 5, 680-687.

35. Ao, A.; Williams, C.H.; Hao, J.; Hong, C.C. Modified Mouse Embryonic Stem Cell based Assay for Quantifying Cardiogenic Induction Efficiency. J. Vis. Exp. 2011, in press.

36. Zhang, X. Modulation of embryonic stem cell fate and somatic cell reprogramming by small molecules. Reprod. Biomed. Online 2010, 21, 26-36.

37. Wang, H.; Hao, J.; Hong, C.C. Cardiac Induction of Embryonic Stem Cells by a Small Molecule Inhibitor of Wnt/ $\beta$-Catenin Signaling. ACS Chem. Biol. 2010, 6, 192-197.

38. Zhu, S.; Wurdak, H.; Wang, J.; Lyssiotis, C.A.; Peters, E.C.; Cho, C.Y.; Wu, X.; Schultz, P.G. A small molecule primes embryonic stem cells for differentiation. Cell Stem Cell 2009, 4, 416-426.

39. Saxe, J.P.; Wu, H.; Kelly, T.K.; Phelps, M.E.; Sun, Y.E.; Kornblum, H.I.; Huang, J. A phenotypic small-molecule screen identifies an orphan ligand-receptor pair that regulates neural stem cell differentiation. Chem. Biol. 2007, 14, 1019-1030.

40. Arrell, D.K.; Terzic, A. Network systems biology for drug discovery. Clin. Pharmacol. Ther. 2010, 88, 120-125.

41. Waring, J.F.; Ciurlionis, R.; Jolly, R.A.; Heindel, M.; Ulrich, R.G. Microarray analysis of hepatotoxins in vitro reveals a correlation between gene expression profiles and mechanisms of toxicity. Toxicol. Lett. 2001, 120, 359-368.

42. Lamb, J.; Crawford, E.D.; Peck, D.; Modell, J.W.; Blat, I.C.; Wrobel, M.J.; Lerner, J.; Brunet, J.P.; Subramanian, A.; Ross, K.N.; et al. The Connectivity Map: using gene-expression signatures to connect small molecules, genes, and disease. Science 2006, 313, 1929-1935.

43. Rho, S.B.; Kim, B.; Kang, S. A gene signature-based approach identifies thioridazine as an inhibitor of phosphatidylinositol-3'-kinase (PI3K)/AKT pathway in ovarian cancer cells. Gynecol. Oncol. 2011, 120, 121-127.

44. Lomenick, B.; Hao, R.; Jonai, N.; Chin, R.M.; Aghajan, M.; Warburton, S.; Wang, J.; Wu, R.P.; Gomez, F.; Loo, J.A.; et al. Target identification using drug affinity responsive target stability (DARTS). Proc. Natl. Acad. Sci. USA 2009, 106, 21984-21989. 
45. Rix, U.; Superti-Furga, G. Target profiling of small molecules by chemical proteomics. Nat. Chem. Biol. 2009, 5, 616-624.

46. Kley, N. Chemical dimerizers and three-hybrid systems: Scanning the proteome for targets of organic small molecules. Chem. Biol. 2004, 11, 599-608.

47. Senderowitz, H.; Marantz, Y.G. Protein-coupled receptors: Target-based in silico screening. Curr. Pharm. Des. 2009, 15, 49-68.

48. Hao, J.; Ho, J.N.; Lewis, J.A.; Karim, K.A.; Daniels, R.N.; Gentry, P.R.; Hopkins, C.R.; Lindsley, C.W.; Hong, C.C. In vivo structure-activity relationship study of dorsomorphin analogues identifies selective VEGF and BMP inhibitors. ACS Chem. Biol. 2010, 5, 245-253.

49. FDA ICH Guidance for industry: S7A Safety Pharmacology Studies for Human Pharmaceuticals:

US Department of Health and Human Services. Available online: http://www.fda.gov/downloads/ Drugs/GuidanceComplianceRegulatoryInformation/Guidances/ucm074959.pdf (accessed on 28 March 2011).

50. Milan, D.J.; Jones, I.L.; Ellinor, P.T.; MacRae, C.A. In vivo recording of adult zebrafish electrocardiogram and assessment of drug-induced QT prolongation. Am. J. Physiol. Heart Circ. Physiol. 2006, 291, H269-H273.

51. Burns, C.G.; Milan, D.J.; Grande, E.J.; Rottbauer, W.; MacRae, C.A.; Fishman, M.C. High-throughput assay for small molecules that modulate zebrafish embryonic heart rate. Nat. Chem. Biol. 2005, 1, 263-264.

52. Schwerte, T.; Pelster, B. Digital motion analysis as a tool for analysing the shape and performance of the circulatory system in transparent animals. J. Exp. Biol. 2000, 203, 1659-1669.

53. Airhart, M.J.; Lee, D.H.; Wilson, T.D.; Miller, B.E.; Miller, M.N.; Skalko, R.G. Movement disorders and neurochemical changes in zebrafish larvae after bath exposure to fluoxetine (PROZAC) Neurotoxicol. Teratol. 2007, 29, 652-664.

54. Richards, F.M.; Kimber, G.; Butler, P.; Waldron, G.; Knight, T.; Goldsmith, P. Fleming, A.L.; Prediction of ophthalmic effects in man by screening drugs for the optokinetic and optomotor responses in zebrafish. Invest. Ophthalmol. Vis. Sci. 2006, 2622, 123.

55. Berghmans, S.; Hunt, J.; Roach, A.; Goldsmith, P. Zebrafish offer the potential for a primary screen to identify a wide variety of potential anticonvulsants. Epilepsy Res. 2007, 75, 18-28.

56. Best, J.D.; Berghmans, S.; Hunt, J.J.; Clarke, S.C.; Fleming, A.; Goldsmith, P.; Roach, A.G. Non-associative learning in larval zebrafish. Neuropsychopharmacology 2008, 33, 1206-1215.

57. Pack, M.; Solnica-Krezel, L.; Malicki, J.; Neuhauss, S.C.; Schier, A.F.; Stemple, D.L.; Driever, W.; Fishman, M.C. Mutations affecting development of zebrafish digestive organs. Development 1996, 123, 321-328.

58. Berghmans, S.; Butler, P.; Goldsmith, P.; Waldron, G.; Gardner, I.; Golder, Z.; Richards, F.M.; Kimber, G.; Roach, A.; Alderton, W.; et al. Zebrafish based assays for the assessment of cardiac, visual and gut function-potential safety screens for early drug discovery. J. Pharmacol. Toxicol. Methods 2008, 58, 59-68.

59. Barrett, R.; Chappell, C.; Quick, M.; Fleming, A. A rapid, high content, in vivo model of glucocorticoid-induced osteoporosis. Biotechnol. J. 2006, 1, 651-655. 
60. Rihel, J.; Prober, D.A.; Arvanites, A.; Lam, K.; Zimmerman, S.; Jang, S.; Haggarty, S.J.; Kokel, D.; Rubin, L.L.; Peterson, R.T.; et al. Zebrafish behavioral profiling links drugs to biological targets and rest/wake regulation. Science 2010, 327, 348-351.

61. Snaar-Jagalska, B.E. ZF-CANCER: Developing high-throughput bioassays for human cancers in zebrafish. Zebrafish 2009, 6, 441-443.

(C) 2011 by the authors; licensee MDPI, Basel, Switzerland. This article is an open access article distributed under the terms and conditions of the Creative Commons Attribution license (http://creativecommons.org/licenses/by/3.0/). 